\title{
Analytical predictions of fragment penetration through hollow concrete masonry units
}

\author{
David Bogosian ${ }^{\mathrm{a}, *}$ and Bence Gerber ${ }^{\mathrm{b}}$ \\ ${ }^{a}$ Karagozian \& Case, 2550 N. Hollywood Way, Suite 500, Burbank, CA 91505, USA \\ ${ }^{\mathrm{b}}$ Century Dynamics, Inc., 1001 Galaxy Way, Suite 325, Concord, CA 94520, USA
}

Received 3 February 2005

Revised 27 December 2006

\begin{abstract}
Modeling steel casing fragment impacts on hollow CMU poses some problems, since the fragments will typically penetrate through the front face and may also penetrate the back face. Techniques are needed for predicting (a) the size of the hole created by the penetration, (b) the size of the annular region of damaged concrete around the hole, and (c) the residual velocity of the fragment. A series of calculations using the AUTODYN code were performed to investigate the accuracy and reliability of the model. The model uses the smooth particle hydrodynamics (SPH) approach to represent the CMU. A variety of steel fragment sizes were projected at a layer of CMU, and the resulting hole size, damage, and fragment residual velocity were tabulated. Results were validated against existing empirical relationships to insure the model's applicability, while additional analyses demonstrated trends and parametric sensitivity.
\end{abstract}

\section{Introduction}

The sphere of military operations is rapidly shifting due to the evolution of asymmetric warfare in the early $21^{\text {st }}$ century. Targets of interest to air operations planners and munitions developers are no longer limited to hardened facilities, whether deeply buried or of massive concrete construction. More and more, there is a need to predict weapons effects on conventional soft targets such as brick and masonry walls, and to develop new munitions and targeting schemes which limit collateral damage and achieve specific objectives.

For this reason, the US Air Force in interested in improving the predictive capabilities of its Munitions Effects/Vulnerability Assessment (MEVA) code used in the study and development of innovative munitions concepts. One of the models currently being developed is a fast-running model to predict the damage to and response of a concrete masonry unit (CMU) wall exposed to the combined blast and fragment effects from a cased weapon detonation. The fast-running model will be based on a suite of high fidelity physics-based (HFPB) analyses using first-principles nonlinear finite element codes.

Modeling airblast loads on CMU walls poses no new or unique challenges. However, modeling fragment effects on such soft targets is problematic in a number of ways. Starting with some seminal experimental studies [1], earlier research has produced a well-accepted and documented methodology for representing fragment loads on reinforced concrete walls by using short duration, high intensity pressure pulses [2]. This approach has then been automated and streamlined for application to typical finite element models of such walls [3].

However, the nature of the interaction between a fragment and a reinforced concrete wall is quite different than that between a fragment and a hollow CMU wall. For typical concrete construction in a hardened bunker, the fragments do not penetrate the entire thickness of the wall. The main features to be predicted are the depth of penetration and,

${ }^{*}$ Corresponding author: David Bogosian, Applied Research Associates, 1055 E. Colorado Blvd., Suite 500, Pasadena, CA 91106, USA. Tel.: +1 626204 4010; E-mail: dbogosian@ara.com. 
more importantly from the aspect of wall response models, the amount of impulse imparted to the wall. And this has in fact been the focus of the research to date as cited above.

For a hollow CMU wall, the fragment is very likely to penetrate at least the front face shell of the unit, and quite possibly also the back face. Not only is CMU material much weaker than typical concrete (by a factor of 2-3), but also the typical face shell thickness of $11 / 4$ inches will prevent penetration of only the smallest of fragments from a typical weapon. While we are still interested in the amount of impulse imparted to the wall, we are much more interested in the damage caused by the penetration event to the wall: the size of the hole produced, and the extent of damaged material left in the wake of the penetration. Finally, we need to predict the residual velocity of the fragment, so that the process may be repeated for the back face interaction.

The ultimate goal is to be able to model the response of a CMU wall in a finite element code to combined blast and fragment effects. Since fully coupled calculations are too time-consuming and complex to generate the hundreds of training points needed for fast-running model generation, the wall model will be decoupled from the load-producing mechanisms. This in turn requires that the damaging effects of fragment impacts be represented in the model, most likely by creating regions in the mesh where the local concrete material properties have been reduced or the elements completely eliminated, to account for the holes made by the fragments. To do this we need a validated HFPB approach for predicting fragment damage and residual velocity. This paper summarizes one such approach which uses the AUTODYN finite element code.

A series of proof-of-concept HFPB calculations were performed for various fragment mass, velocity, and impact angle values, and also for various CMU shell thicknesses and strengths. The objective of the calculations was to analytically replicate fragment impact onto a CMU shell to measure certain key metrics: (a) penetration, (b) fragment residual velocity, (c) size of the damaged region or hole in the CMU, and (d) momentum imparted to the CMU.

Since there is no experimental data against which to validate the calculations, a limited validation was performed against existing empirical methods. This was done within the parametric range of applicability of the empirical methods, which unfortunately does not coincide with the range of interest for the CMU problem. However, once the applicability of the analytical method was demonstrated, the HFPB method was then applied with greater confidence within the parameter range of interest.

\section{Analytical methods}

The HFPB model used in this case was AUTODYN; while the code is capable of 3D problems, this particular problem was run in 2D axisymmetric mode, since the fragment impact angle was normal to the plane of the CMU. AUTODYN is a non-linear commercially available code with multiple solution techniques which can be used individually or in combination. These include Euler, Lagrange, adaptive Lagrangian-Eulerian (ALE), and the mesh-free smooth particle hydrodynamics (SPH) technique [4]. AUTODYN has been used for several previous impact calculations which were validated against test data [5,6].

SPH is a gridless Lagrangian technique that originated in 1977. The main advantage of the method is to bypass the requirement for a numerical grid to calculate spatial derivatives. This avoids the severe problems associated with mesh tangling and distortion which usually occur in Lagrangian analyses involving large deformation impact and explosive loading events. Although the name for the technology includes the term 'Hydrodynamic', thereby reflecting its original basis in hydrodynamic applications, in fact material strength can be included [7].

Grid based methods, such as Lagrange and Euler, assume a connectivity between nodes to construct spatial derivatives. SPH uses a kernel approximation, which is based on randomly distributed interpolation points with no assumptions about which points are neighbors, to calculate spatial derivatives. To illustrate this consider a continuum represented by a set of interacting particles, as shown in Fig. 1. Each particle "I" interacts with all other particles "J" that are within a given distance (usually assumed to be $2 \mathrm{~h}$ ) from it. The distance $\mathrm{h}$ is called the smoothing length. The interaction is weighted by the function $W\left(\mathbf{x}-\mathbf{x}^{\prime}, h\right)$ which is called the smoothing (or kernel) function. Using this principal, the value of a continuous function, or its derivative, can be estimated at any particle "I" based on known values at the surrounding particles " $\mathrm{J}$ " using the following kernel estimates: 

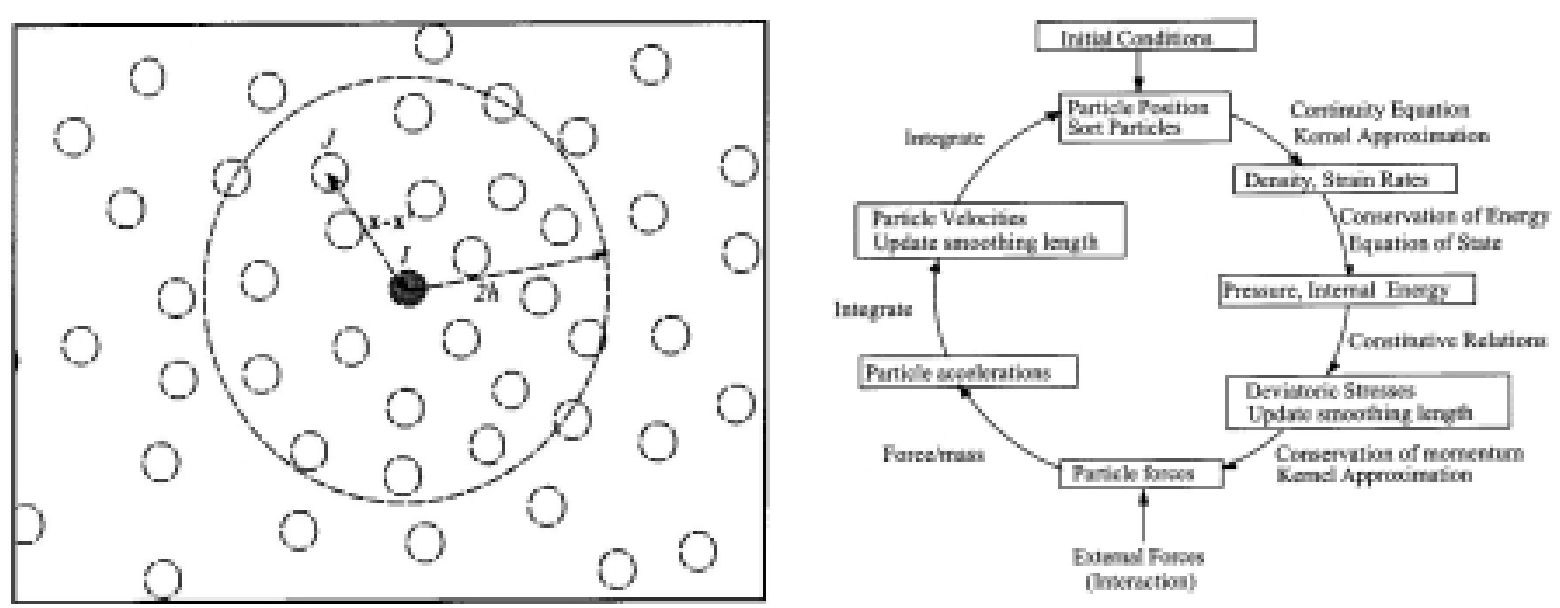

Fig. 1. Neighboring particle geometry and computational cycle for SPH methodology.

$$
\begin{gathered}
f(\mathbf{x}) \approx \int f\left(\mathbf{x}^{\prime}\right) W\left(\mathbf{x}-\mathbf{x}^{\prime}, h\right) d \mathbf{x}^{\prime} \\
\nabla \cdot f(\mathbf{x}) \approx \int \nabla \cdot f\left(\mathbf{x}^{\prime}\right) W\left(\mathbf{x}-\mathbf{x}^{\prime}, h\right) d \mathbf{x}^{\prime}
\end{gathered}
$$

where $f$ is a function of the three-dimensional position vector $\mathbf{x}$ and $d \mathbf{x}^{\prime}$ is a volume.

After several steps of derivation and by converting the continuous volume integrals to sums over discrete interpolation points the equations can be expressed in several forms, with commonly used symmetric formulations for the gradient being:

$$
\begin{aligned}
& \nabla \cdot f\left(\mathbf{x}^{I}\right) \approx \frac{1}{\rho^{I}} \sum_{J=1}^{N} m^{J}\left(f\left(\mathbf{x}^{I}\right)-f\left(\mathbf{x}^{J}\right)\right) \cdot \nabla W\left(\mathbf{x}^{I}-\mathbf{x}^{J}, h\right) \\
& \nabla \cdot f\left(\mathbf{x}^{I}\right)=-\rho^{I} \sum_{J=1}^{N} m^{J}\left(\frac{f\left(\mathbf{x}^{I}\right)}{\left(\rho^{I}\right)^{2}}+\frac{f\left(\mathbf{x}^{J}\right)}{\left(\rho^{J}\right)^{2}}\right) \cdot \nabla W\left(\mathbf{x}^{I}-\mathbf{x}^{J}, h\right)
\end{aligned}
$$

where the gradient $\nabla W$ is with respect to $\mathbf{x}^{J}, m$ is the mass, and $\rho$ is the density.

In SPH, the basic steps used in each calculation cycle, as implemented in AUTODYN-2D, are shown in Fig. 2. The calculation cycle is similar to that for a Lagrange zone, except for the steps where a kernel approximation is used. Kernel approximations are used to compute forces from spatial derivatives of stress and spatial derivatives of velocity are required to compute strain rates. In addition SPH requires a sort of the particles at least once every cycle in order to locate current neighboring particles. When variable smoothing is used ( $h$ can vary locally in space and time) the smoothing length is updated twice per cycle.

\section{Analytical model}

The particular model used for this problem (Fig. 2) used a Lagrangian mesh for the fragment (assumed to be spherical) and an SPH mesh for the CMU. The fragment was assumed to be rigid, while realistic concrete properties (for a low-strength, 2,000 psi concrete) were used for the CMU. Figure 3 shows a close-up of the fragment and CMU meshes, illustrating the particle density used in the SPH mesh for the CMU.

To exercise the model, some typical fragment masses and velocities were chosen which are more or less representative of the range of values of interest. These include variability not only in the weapon parameters but also the expected mass distribution of the fragments produced. 


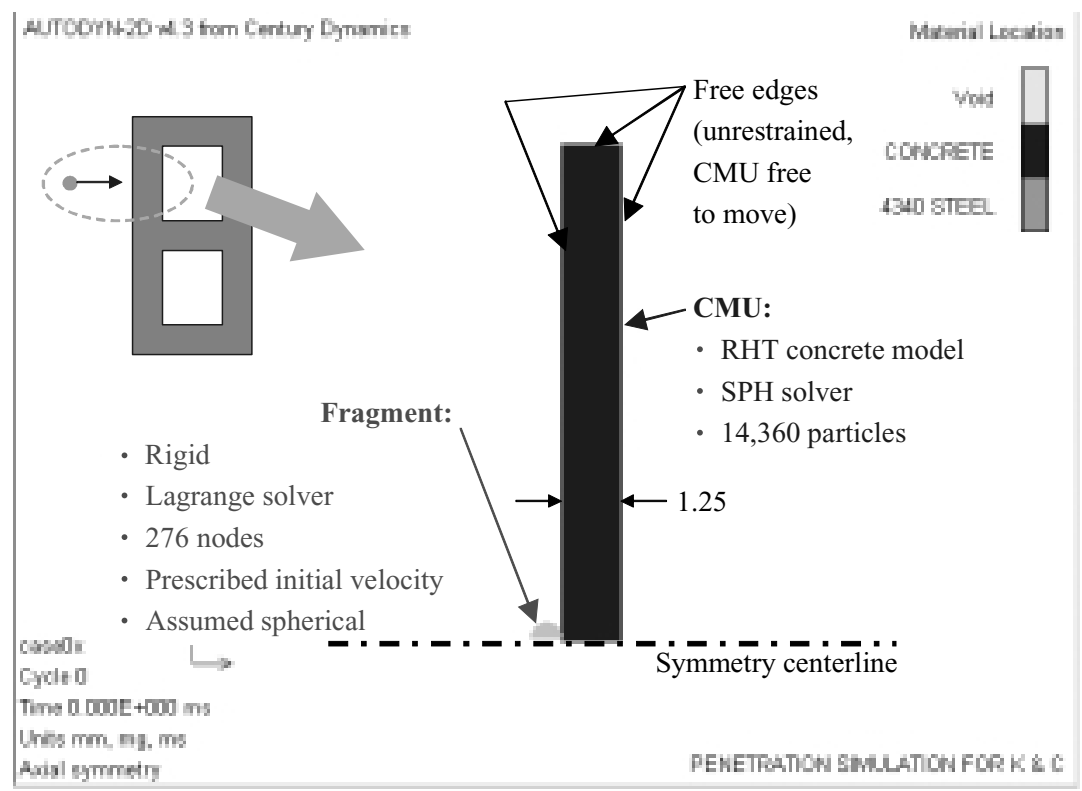

Fig. 2. Overview of model used to calculate fragment penetration of CMU.

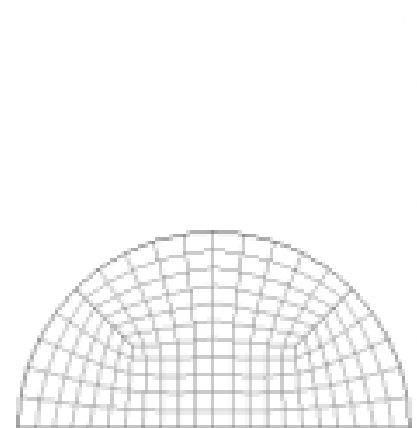

Fragment

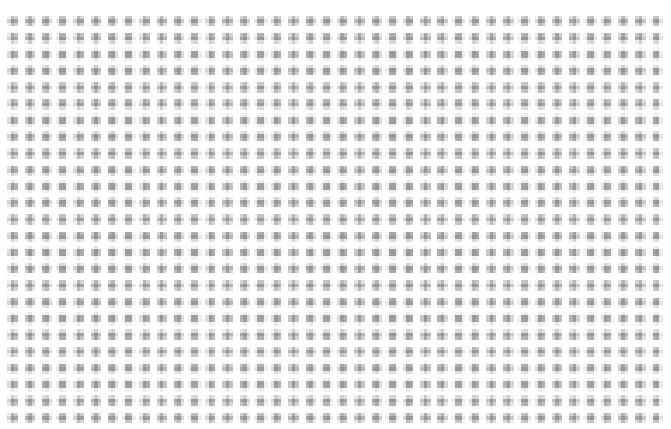

CMU

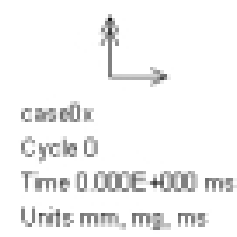

PENETRATION SIMULATION FOR K \& C

Fig. 3. Close-up view of mesh representing fragment and CMU.

\section{Analytical calculations}

Three sets of calculations were made. The initial set used parameters within the applicability limits of the empirical method, in order to gain confidence in the validity of the AUTODYN model. Once that was demonstrated, a second set was calculated for parameters more directly relevant to CMU materials. Finally two calculations were made to investigate sensitivity of the results to variations in the analytical model. A summary of these calculations is shown in Table 1. 
Table 1

Parameters of AUTODYN fragment impact calculations

\begin{tabular}{lrlcc}
\hline & Run & $\begin{array}{l}\text { Calculation } \\
\text { description }\end{array}$ & $\begin{array}{c}\text { Fragment } \\
\text { mass }(\mathrm{g})\end{array}$ & $\begin{array}{c}\text { Fragment } \\
\text { velocity (m/s) }\end{array}$ \\
\hline Validation & 3 & Rigid frag, SPH, $f_{c}^{\prime}=4000$ & 7.9 & 1680 \\
Parameter Study & 4 & Rigid frag, SPH, $f_{c}^{\prime}=4000$ & 142 & 1680 \\
& 5 & Rigid frag, SPH, $f_{c}^{\prime}=2000$ & 1.5 & 1370 \\
Sensitivity & 6 & Rigid frag, SPH, $f_{c}^{\prime}=2000$ & 17.7 & 1370 \\
& 7 & Rigid frag, SPH, $f_{c}^{\prime}=2000$ & 28.6 & 1370 \\
& 10 & Frag $f_{y}=140 \mathrm{ksi}, \mathrm{SPH}, f_{c}^{\prime}=2000$ & 28.6 & 1370 \\
& 2 & Rigid frag, Lagrange, $f_{c}^{\prime}=4000$ & 7.9 & 1680 \\
\hline
\end{tabular}
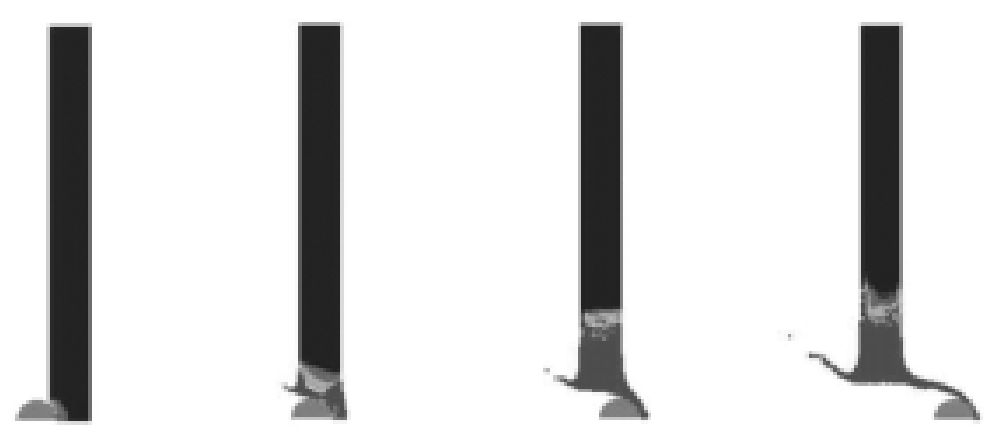

Fig. 4. Snapshots from analysis of $142 \mathrm{~g}$ fragment impact on CMU shell.

\section{Calculations for validation against empirical method}

The first set of calculations was used to compare against the results of an established empirical methodology comprised of a set of equations that estimate penetration depth and residual velocity for a fragment impacting a layer of concrete. The equations take as their inputs the fragment mass $m_{f}$, the fragment impact velocity $V_{s}$, the fragment aspect ratio $(l / d)$, and the concrete strength $f_{c}^{\prime}$. Given these inputs, one may compute the thickness required to prevent penetration $T_{p}$ and the residual velocity $V_{r}$.

The methodology is limited in its application to fragments between 6 and $460 \mathrm{~g}$, and for concrete with strength between 4,000 and 6,000 psi. Although CMU is typically around 1,500 psi in strength, the AUTODYN validation analyses were performed for 4,000 psi concrete to allow a full comparison against the empirical method.

Two cases were analyzed, in which $7.9 \mathrm{~g}$ and $142 \mathrm{~g}$ fragments impacted a 4,000 psi concrete shell at 1,680 m/s. Both penetrated through the concrete shell. Figure 4 shows a series of snapshots during the penetration, showing the concrete damage (in red) and the production of a hole in the concrete as well as the concrete debris being ejected off the back face.

Two such calculations were performed using AUTODYN, one for the $7.9 \mathrm{~g}$ and one for the $142 \mathrm{~g}$ fragments. The results, in terms of fragment residual velocity, are compared against those predicted by the empirical method in Fig. 5 for a broad spectrum of masses, including those that are below the limit of applicability of the method $(6 \mathrm{~g})$ but were nevertheless calculated out of curiosity. We note that the AUTODYN model is low by 10-15\%, but this is quite reasonable in light of the empiricism of the method as well as the wide uncertainty bands associated with the predictions. It is encouraging to note that the slopes of the data from both AUTODYN and DAHS are very similar, and that as the mass is increased, the residual velocity approaches the initial impact velocity as would be expected. Thus, we conclude that the AUTODYN model produces reasonable results within the realm of applicability of the empirical method, which allows us to perform further calculations for lower strength concrete in the range of CMU strengths. 


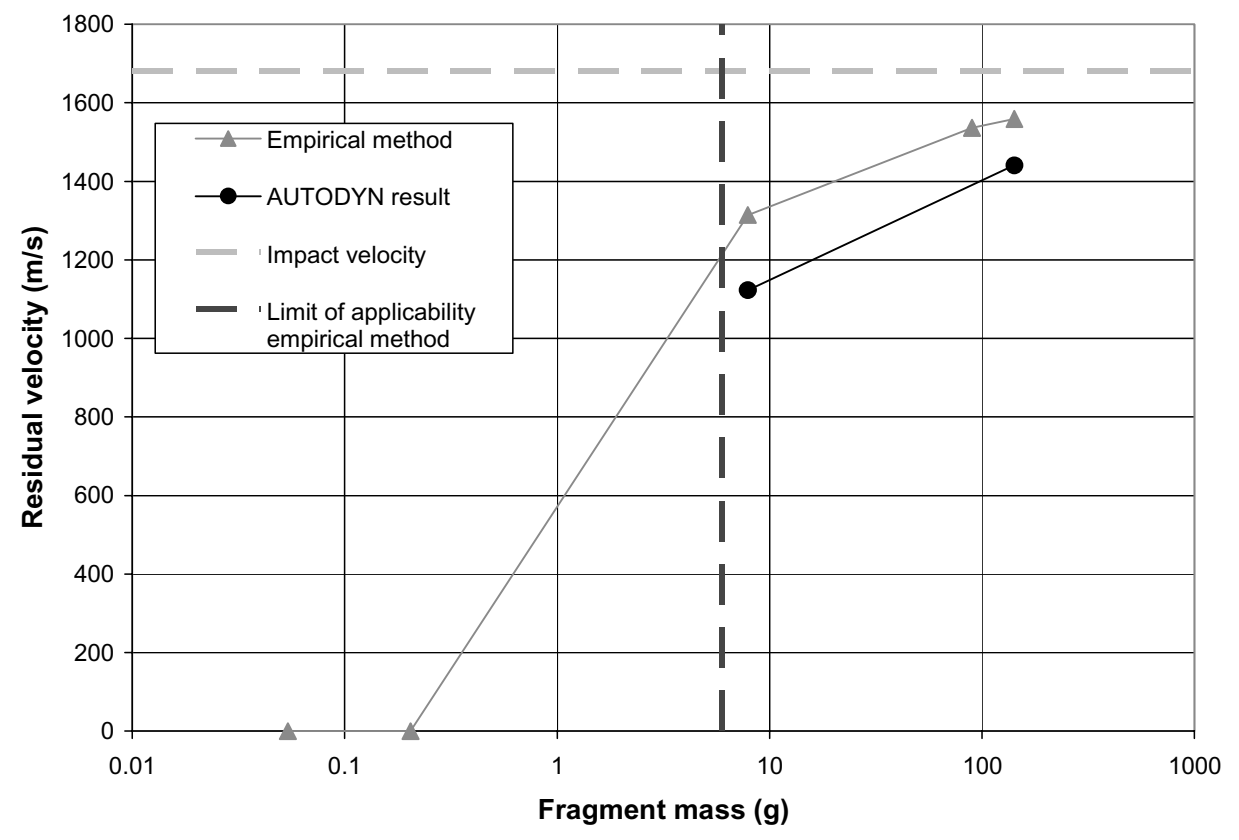

Fig. 5. Comparison of AUTODYN results against empirical methodology for 4000 psi concrete target.

\section{Calculations for realistic CMU strength}

Three calculations were performed using 2,000 psi concrete and with 1.5, 17.7, and $26.6 \mathrm{~g}$ fragments (runs 5-7 in Table 1). All the fragments penetrated the concrete and produced the residual velocity trend shown in Fig. 6, which is reasonable; results from the same empirical method (albeit outside the published domain of application) are shown for comparison. The final damage plots are shown in Fig. 7. In each case, the fragment creates a hole but also creates a region of completely failed concrete (in red) which has essentially lost any cohesive strength and has sand-like properties. Thus, we may assume that this entire region will be part of the "hole" in the final post-penetration condition.

To estimate the size of damaged area in the CMU shell produced by the impact, various techniques are possible. For instance (Fig. 8), one may take the innermost radius at which the entire thickness has reached full damage $\left(R_{1}\right)$, or the outermost radius at which any portion of the thickness has reached full damage $\left(R_{2}\right)$. For the purposes of this study, $R_{1}$ was utilized, but using the average of $R_{1}$ and $R_{2}$ would be equally representative of a hole diameter. Fortunately, the model indicates that the difference between $R_{1}$ and $R_{2}$ is rather insignificant, which in turn suggests that the annular region of partially damaged but not completely failed concrete will be very small.

Taking the radius of the fully red zone $\left(R_{1}\right)$ from both the initial validation and these calculations, and plotting it against the mass of the fragment produces the plot in Fig. 9, which suggests that the hole diameter is simply correlated to mass and independent of velocity. This hypothesis will require further validation through additional calculations using other fragment masses and velocities and concrete strengths. However, if it is tenable, that would result in a significant and important simplification of the final model. If hole diameter is simply related to fragment mass, parameters such as velocity and concrete strength can be eliminated, which reduces the number of analyses required to formulate a general model.

Very limited experimental data is available for fragment penetration into hollow CMU, and it consists essentially of post-test photographic images (Fig. 10) that show the damage caused by fragments from a small mortar round detonation [8]. Yet, qualitative as the data might be, it indicates that the analytical results from AUTODYN are reasonable. The fragments produce relatively small round holes (ranging in size from 1 to 5 inches in diameter), as predicted by AUTODYN. And the holes appear to be "clean" without any obvious signs of an annular region of damaged concrete, although a detailed examination of the holes is not possible from these photographs. 


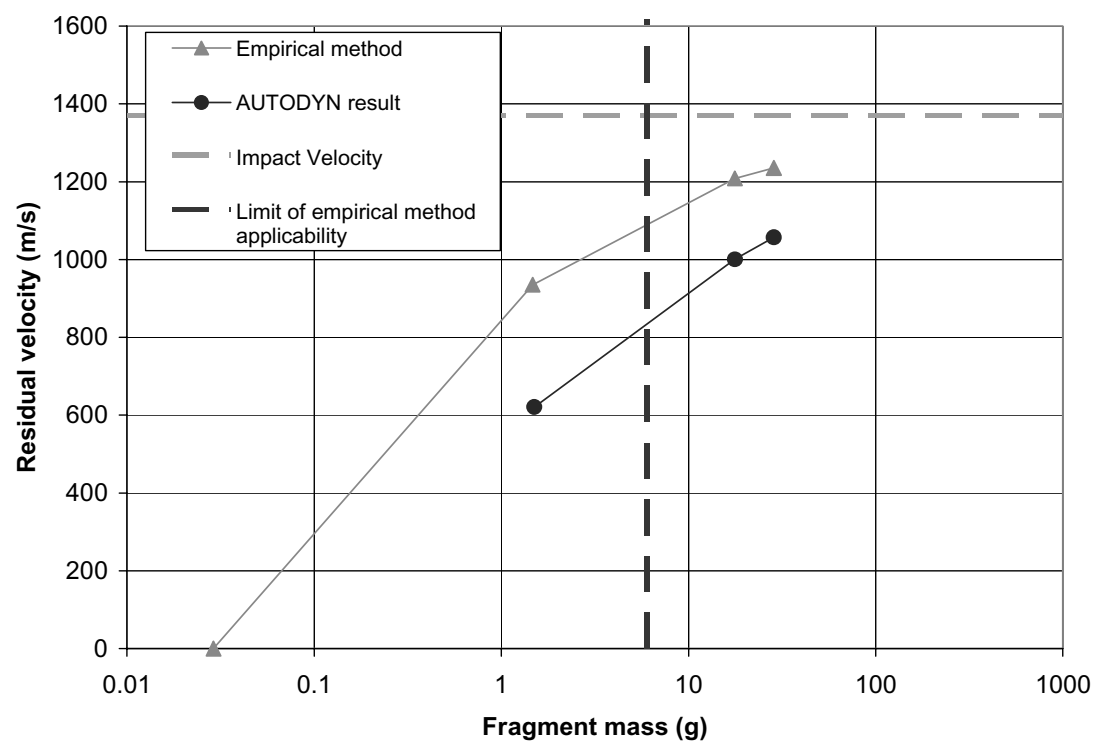

Fig. 6. Residual velocity for fragments impacting 2,000 psi concrete shell.

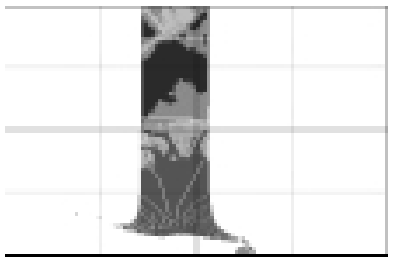

(a) $\operatorname{mass}=1.5 \mathrm{~g}$

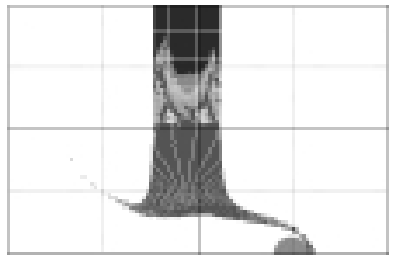

(b) $\operatorname{mass}=17.7 \mathrm{~g}$

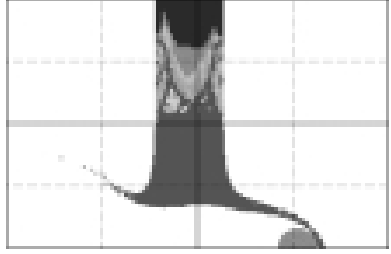

(c) $\operatorname{mass}=28.6 \mathrm{~g}$

Fig. 7. Final damage states from fragment impacts at $1,370 \mathrm{~m} / \mathrm{s}$ on 2000 psi targets.

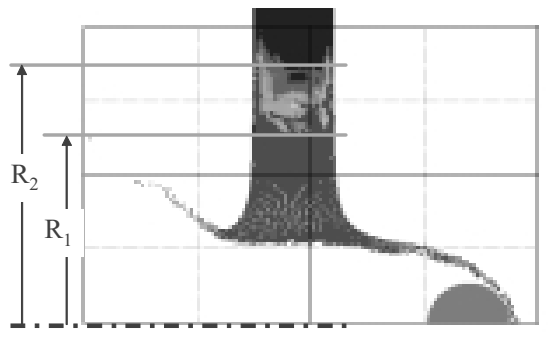

Fig. 8. Radii to be used in estimating damaged region of CMU.

Other test data has been identified from experiments where fragments were projected at hollow CMU and their residual velocity measured [9]. Unfortunately, in these tests the fragments penetrated both front and back shells, and the residual measurements were taken after both events, so that the residual velocity after a single penetration event cannot be directly extracted.

Even so, we may assume that the kinetic energy lost during each of the two penetration events (front and back face shell) is equal. With this assumption, we can calculate the residual velocity after the first penetration $\left(V_{\text {res }}\right)$ as a function of the initial velocity $V_{o}$ and the final residual velocity $V_{f}$ as follows:

$$
V_{\text {res }}=\sqrt{2\left(V_{o}^{2}+V_{f}^{2}\right)}
$$




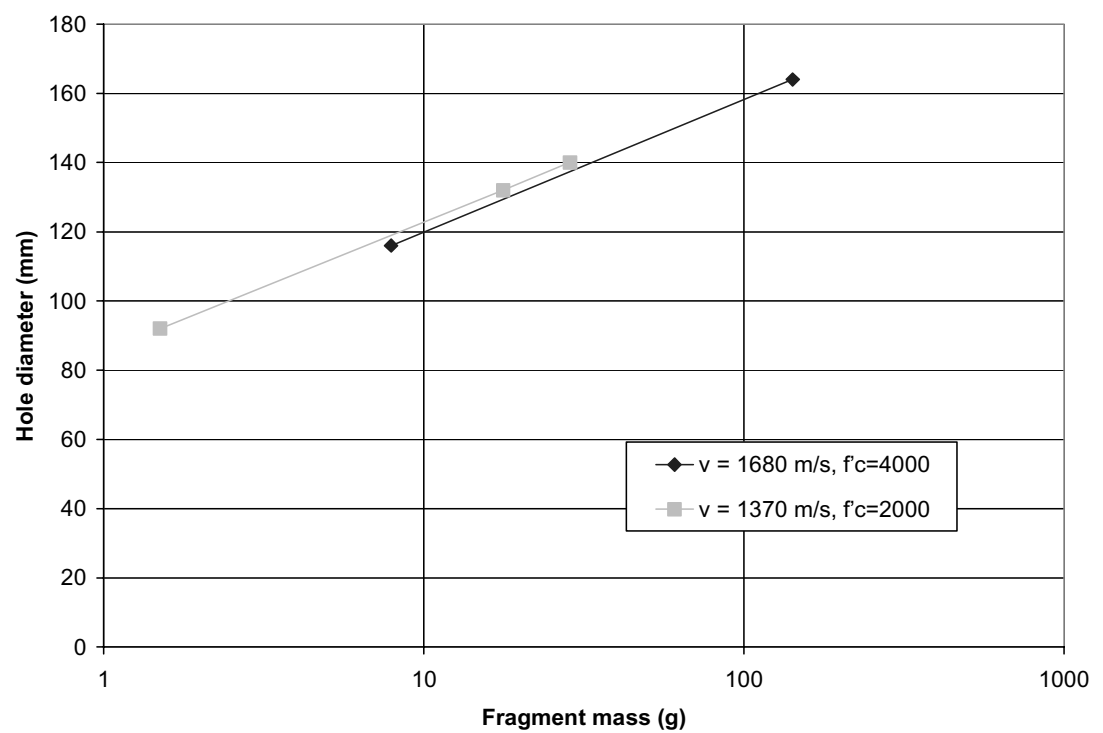

Fig. 9. Hole diameter as a function of fragment mass from AUTODYN calculations.

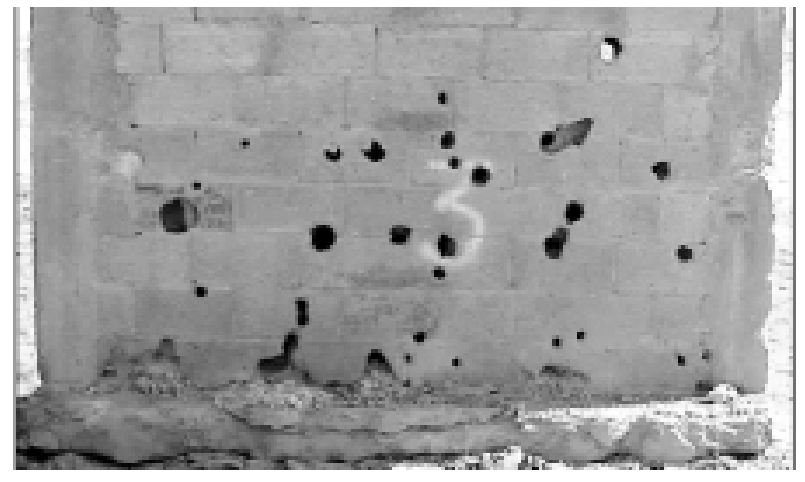

Fig. 10. Fragment-induced damage to hollow CMU wall from mortar round detonation.

Since these tests were conducted for other fragment mass and velocity combinations, it is helpful to consider the ratio of initial velocity to residual velocity as a way of normalizing the data and facilitating comparisons. Figure 11 indicates that, on this basis, and under the assumption of equal energy loss in each of the two penetration events, the test data compare very well with those produced by AUTODYN.

\section{Sensitivity studies}

Two additional calculations were made as sensitivity studies to assess the effect on the results of varying certain assumptions in the analysis. The first (run 10 in Table 1) involved a realistic deformable fragment as opposed to the rigid assumption made in all the previous calculations. A typical $140 \mathrm{ksi}$ strength was used for the steel, and the other parameters were set to the same values as run 7 for direct comparison.

The final deformed state of the mesh from both these calculations is displayed in Fig. 12. The deformable fragment has certainly been flattened at its upstream side, but in other respects the two images are quite similar. In fact, the estimated damaged area diameter is identical from both calculations. The residual velocity is slightly lower: $916 \mathrm{~m} / \mathrm{s}$ instead of $1057 \mathrm{~m} / \mathrm{s}$ for the rigid fragment. While the difference is physically reasonable (a deforming fragment will increase in its projected area, creating greater resistance and energy loss), the magnitude of this difference is 


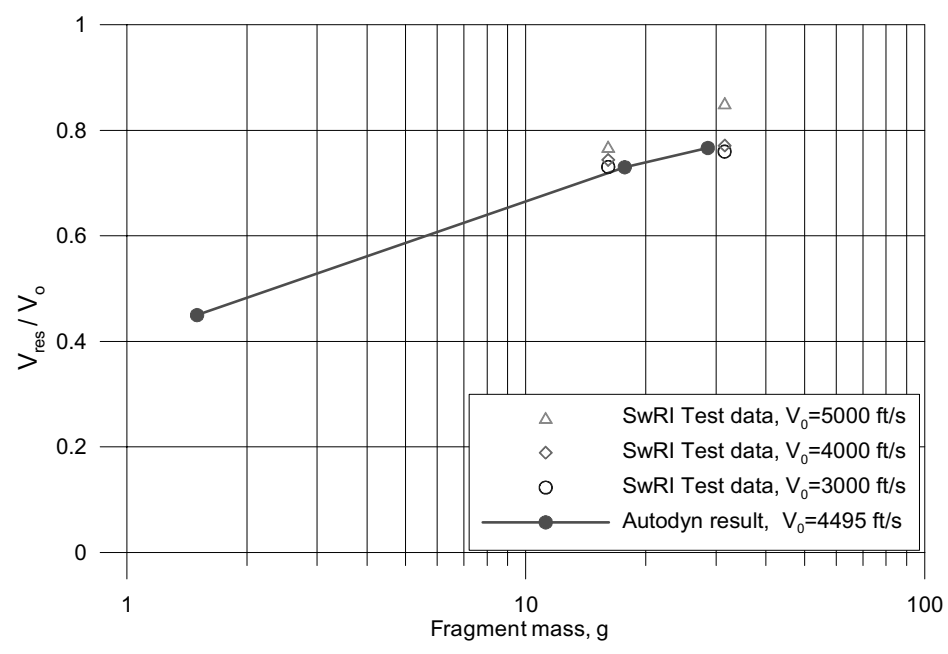

Fig. 11. Residual velocity (as fraction of original velocity) compared to test data.
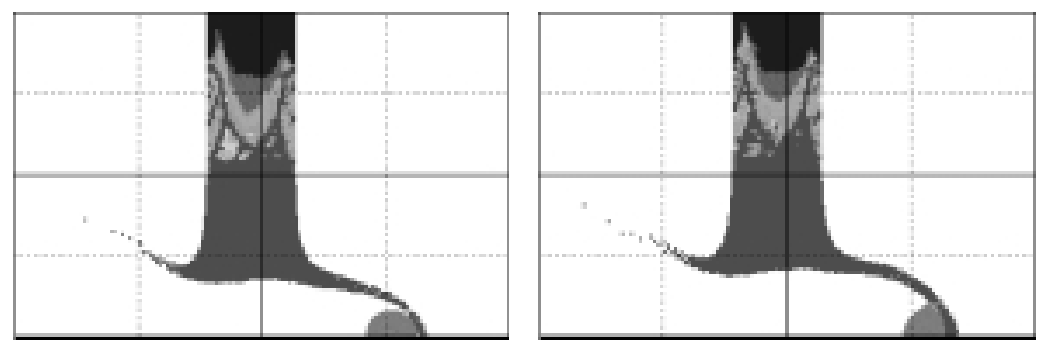

Fig. 12. Comparison of final state from comparable analyses with rigid fragment (left) and deformable fragment (right); in both analyses, $m=$ $28.6 \mathrm{~g}, v=1,370 \mathrm{~m} / \mathrm{s}$.

rather small. It thus appears that the deformability of the fragment is not an important parameter and need not be considered in future studies, unless subsequent experimental results indicate that it is.

The other sensitivity study was performed by varying the analytical method used to calculate the response of the CMU shell from SPH to a Lagrangian formulation that utilizes erosion to eliminate highly damaged elements. This calculation (run 2 in Table 1) was made using the same parameters as run 3 for direct comparison. The difference in residual velocities was completely negligible, $1102 \mathrm{~m} / \mathrm{s}$ instead of $1123 \mathrm{~m} / \mathrm{s}$, and the hole diameters were again identical. Thus there is no apparent advantage to using Lagrange with erosion as opposed to SPH to perform these calculations.

\section{Conclusions}

The analyses described above demonstrate that the current state of the art in non-linear impact mechanics modeling is capable of characterizing the interaction between a metal casing fragment and the shell of a CMU. The results agree well with those from established empirical methods when the model is exercised within the domain of applicability of the empirical method. Outside that domain and in a region of the parameter space that is more representative of typical CMU strengths, the model continues to exhibit reasonable behavior and trends, both in terms of residual velocity and hole diameter.

The results also indicate that the damaged area of concrete is fairly well circumscribed, which would lead to the expectation of a relatively clean, well-defined hole without a large surrounding area of partially damaged concrete. This greatly simplifies the process for creating finite element models of the damaged CMU wall: only the hole need be modeled, without the need for modeling an annular region of partially damaged concrete around it. 
The calculations indicate other noteworthy trends. In general, the amount of concrete material from the front shell being ejected towards the back shell is small, and its momentum negligible relative to that of the fragment itself. This indicates that, with regard to the modeling of CMU wall response, this aspect of the physics is not of primary importance and may be neglected without significantly affecting the results. Similarly, the momentum imparted to the CMU itself as a result of the penetration is very small and also can be neglected. This is certainly true for the more energetic fragments which easily penetrate the face shell, but this assumption will have to be revisited for smaller fragments which may not penetrate as easily or even at all.

All these conclusions remain tentative until high fidelity experiments are conducted to verify that the actual behavior of CMU is similar to that observed in the calculations. Currently available test data is of insufficient detail to allow full validation, but is very encouraging both in terms of the qualitative indications regarding damage to the $\mathrm{CMU}$, and also in terms of the residual velocity after the penetration.

\section{Acknowledgments}

Funding for the research presented in this paper was provided by the Air Force Research Laboratory (AFRL), Munitions Directorate, Lethality and Vulnerability Branch, with Mr. Norman Gagnon serving as the project manager and technical director. The authors gratefully acknowledge Mr. Gagnon's leadership and oversight of this project.

\section{Disclaimer}

The opinions expressed in this paper are exclusively those of the authors. While the research discussed above was funded by the US Government, this should not be construed to imply US Government endorsement of any products or methods.

\section{References}

[1] F.D. Dallriva and J.L. Davis, Multiple-Fragment Impact Experiments on Reinforced Concrete Slabs, US Army Engineer Research and Development Center, technical report ERDC/GSL TR-02-7, June 2002.

[2] C.K.B. Lee, Step-by-step Instructions for Correcting Calculated Transferred Momentum from Fragment Impacts in Coarse-zoned Hydrocode Calculations, CWE Fragment Working Group, April 1997.

[3] D.D. Bogosian and D.A. Simons, An automated methodology for defining fragments from generic cased weapons, Critical Technologies in Shock and Vibration 2(1) (November 2002), 27-35.

[4] C.J. Hayhurst and R.A. Clegg, Cylindrically Symmetric SPH Simulations of Hypervelocity Impacts on Thin Plates, Proceedings of the Hypervelocity Impact Symposium, Freiburg, Germany, October 1996.

[5] K. Thoma, W. Riedel and S. Hiermaier, Mesomechanical Modeling of Concrete Shock Response, Experiments and Linking to Macromechanics by Numerical Analysis, ECCM '99, Munchen, Germany, 1999.

[6] O.F.J. Meuric et al., Numerical Prediction of Penetration into Reinforced Concrete using a Combined Grid based and Meshless Lagrangian Approach, 10th International Symposium on the Interaction of Effects of Munitions with Structures, May 7-11, 2001.

[7] L.D. Libersky et al., High strain Lagrangian hydrodynamics, Journal of Comp Physics 109(1) (November 1993).

[8] R.J. Dinan, D.R. Coltharp and T. Townsend, Fragment Penetration of Conventional Walls with Retrofit Modifications, US Army Corps of Engineers Engineer Research and Development Center, Vicksburg, MS, January 2001.

[9] P.K. Bowles, D.J. Grosch, D. Goodlin and B.T. Plenge, Fragment Penetration Effects Model, Southwest Research Institute, San Antonio, TX, and Air Force Research Laboratory, Eglin AFB, AFRL-MN-EG-TR-1998-7029, March 1998. 

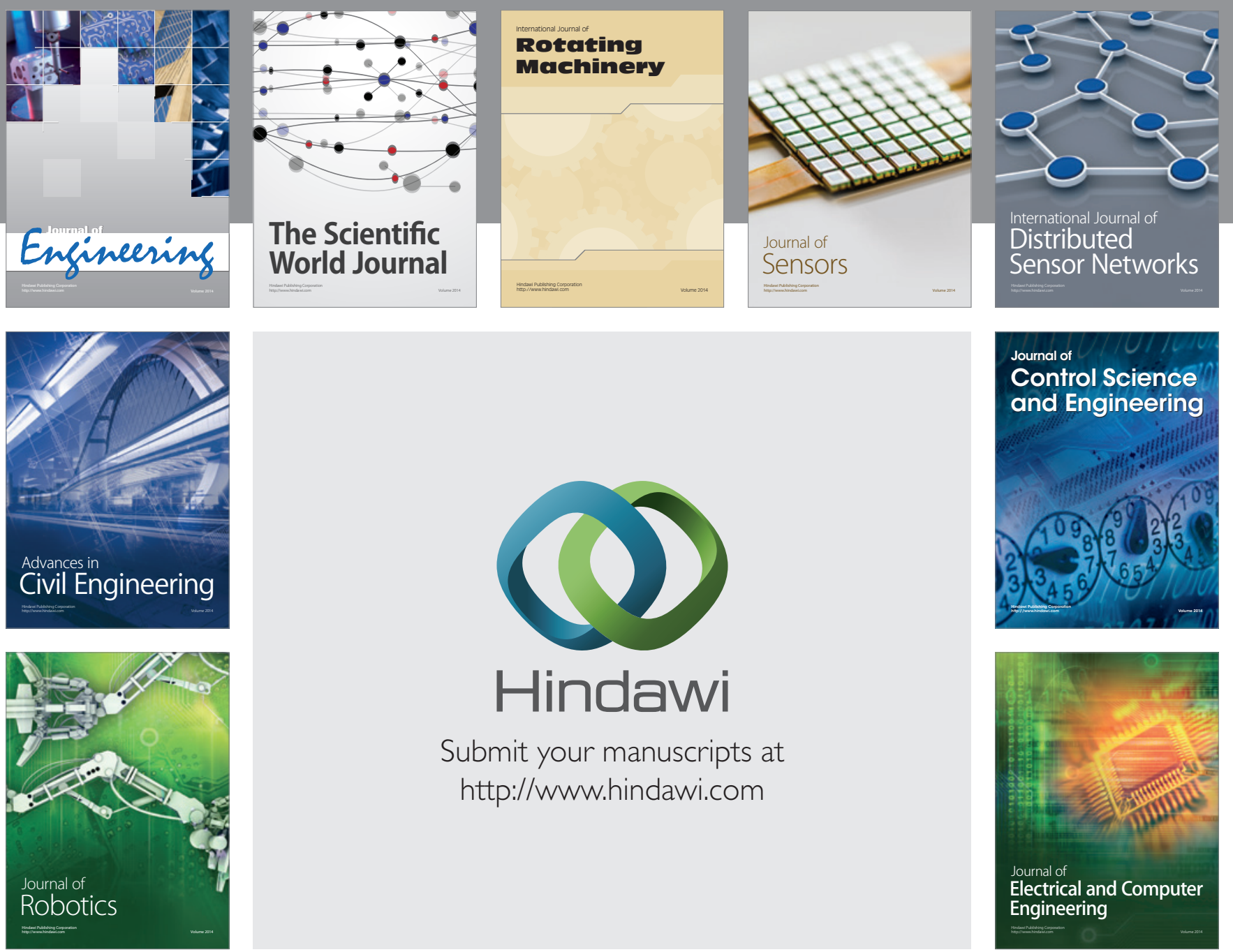

Submit your manuscripts at

http://www.hindawi.com
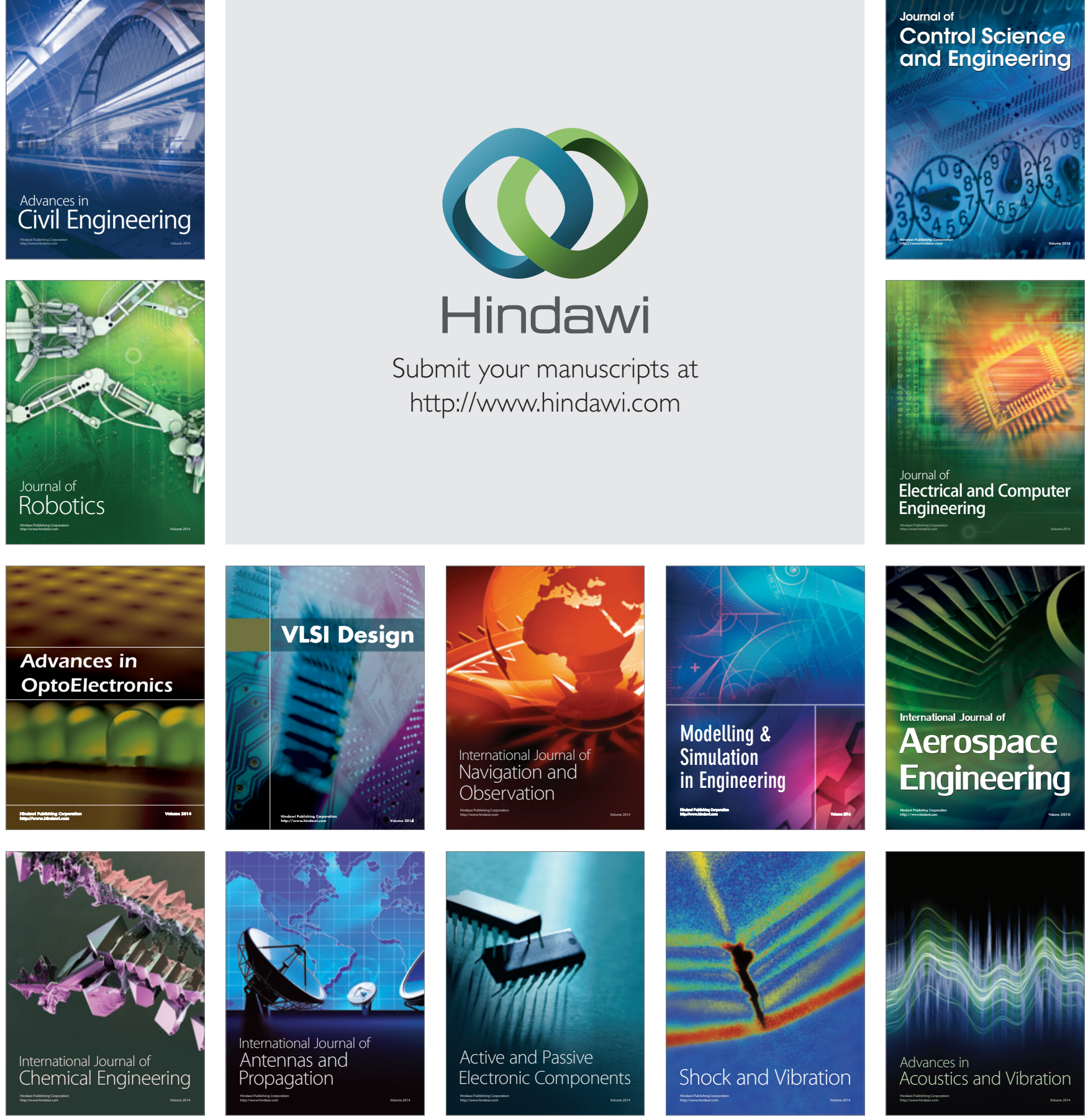